\title{
Surgical footprints, then and now: response to comment by Dietz
}

\author{
Andri Nieuwoudt
}

Received: 26 November 2008 / Accepted: 27 November 2008/Published online: 9 April 2009

(C) The International Urogynecological Association 2009

\section{Dear Editor,}

It's always heart-warming to receive a response to what you write [1] - be it positive or negative. Especially from prominent figures in the field. I think that Professor Dietz [2] and I speak the same language, but on different levels. He refers to the pathogenesis of prolapse, I to the surgical options available to rectify the damage and the imprints these operations leave in the pelvis.

The purpose of my editorial was to highlight the damage that different approaches to pelvic organ reconstructive surgery can do. The focus of the classical anterior repair is to reduce the bulging anterior wall directly by plicating the support tissue in the midline. The focus of paravaginal repairs is on reducing the bulging anterior wall indirectly by attaching the bladder base to the pelvic sidewalls. I think that Prof. Dietz agrees, and in fact he has proved already with his imaging studies ( and even with his educated index finger study in a more recent issue of the journal [3]), that the pathology is lateral and not central. This is enough proof to me that when one performs a central plicating procedure the strain put on the weakened sidewall (be it due to levator muscle damage or to supportive collagen damage) can in due course lead to a recurrence. By focussing on the lateral sidewall and strengthening the support here the damage can be undone.

To go back to the editorial: the main purpose of the reconstructive surgeon is to reconstruct. The new kids on the block, namely the "mesh kits" are all based on paravaginally fixing a bridge for the bulging anterior wall. To

A. Nieuwoudt $(\square)$

Gynaecology, Ziekenhuis Zorgzaam,

Wielingenlaan 2,

4535 PA Terneuzen, The Netherlands

e-mail: nieuwoudt@gmail.com do this one has to break down the lateral supports of the bladder base - in a sense causing a "paravaginal defect". The damage done with this type of surgery is tremendous. To remove these synthetic bridges in the advent of complications leads to further damage, and an added dilemma is how to support the bladder in the secondary procedure. Sounds very destructive to me. To put a permanent mesh between two hollow organs goes against all surgical principles. This is why I want to highlight that this route is not the path to take. As an alternative to - what I see as the discredited concept of - central plications this is not the answer.

A third option is overlooked, namely building a lateral support for the bladder base and indirectly reducing the bulge of the anterior wall, plus improving the collagen quality of the support. Clearly the damage caused by the surgeon is less than the above alternatives.

Maybe this is one of the reasons why there is "confusion in the field of prolapse repair".

These were the surgical footprints to which I referred.

I can assure Prof. Dietz that I do not "propagate rumour and hearsay" - although I had my training in the sphere of the "confusion of the 1970s and 1980s", as I am sure he had!

As they say: watch this space.

\section{References}

1. Nieuwoudt A (2008) Surgical footprints, then and now. Int Urogynecol J 19:1187-1188. doi:10.1007/s00192-008-0685-9

2. Dietz HP (2008) Comment on editorial by Nieuwoudt: "Surgical footprints, then and now". Int Urogynecol J . doi:10.1007/s00192008-0785-6

3. Dietz HP, Shek KL (2008) The quantification of levator muscle resting tone by digital assessment. Int Urogynecol J 19:1489-1493. doi:10.1007/s00192-008-0682-z 